Article

www.mdpi.com/journal/jlpea

\title{
Reconfigurable RF Energy Harvester with Customized Differential PCB Antenna
}

\author{
Alessandro Bertacchini ${ }^{1, \dagger, *}$, Luca Larcher ${ }^{1, \dagger}$, Moreno Maini ${ }^{2, \dagger}$, Luca Vincetti ${ }^{2, \dagger}$ \\ and Stefano Scorcioni ${ }^{3, \dagger}$
}

1 Department of Sciences and Methods for Engineering - DISMI-University of Modena and Reggio Emilia, via G.Amendola, 2, 42122 Reggio Emilia, Italy; E-Mail: luca.larcher@unimore.it

2 Department of Engineering "Enzo Ferrari" — DIEF - University of Modena and Reggio Emilia, via P. Vivarelli, 10/1, 41125 Modena, Italy;

E-Mails: moreno.maini@unimore.it (M.M.); luca.vincetti@unimore.it (L.V.)

3 ASK Industries, via P. Togliatti 46/a 42020 Montecavolo (RE), Italy;

E-Mail: scorcioni.stefano@gmail.com

$\dagger$ These authors contributed equally to this work.

* Author to whom correspondence should be addressed; E-Mail: alessandro.bertacchini@unimore.it; Tel.: +39-0522-522646; Fax: +39-0522-522609.

Academic Editor: Domenico Zito

Received: 24 June 2015 / Accepted: 24 November 2015 / Published: 27 November 2015

\begin{abstract}
In this work, a Radio Frequency (RF) Energy Harvester comprised of a differential Radio Frequency-to-Direct Current (RF-DC) converter realized in ST130 nm Complementary Metal-Oxide-Semiconductor (CMOS) technology and a customized broadband Printed Circuit Board (PCB) antenna with inductive coupling feeding is presented. Experimental results show that the system can work with different carrier frequencies and thanks to its reconfigurable architecture the proposed converter is able to provide a regulated output voltage of $2 \mathrm{~V}$ over a $14 \mathrm{~dB}$ of RF input power range. The conversion efficiency of the whole system peaks at $18 \%$ under normal outdoor working conditions.
\end{abstract}

Keywords: RF energy harvester; reconfigurable RF-DC converter; differential antenna 


\section{Introduction}

Energy harvesting has become an attractive way to power wirelessly connected electronic systems such as sensors without using batteries or other energy source with limited lifetime. The research in this field has been very intense in the last years, and solutions harvesting energy from a large set of sources such as solar light, thermal gradient, strain, vibrations, electromagnetic field, air flow, pressure variations, have been proposed.

Among the various options, the idea of exploiting the background RF signals as a power source for wireless devices is very attractive, especially due to the pervasive diffusion of RF signals used in modern communication systems. Unfortunately, the power associated with communication signals is unpredictable and typically very small, thus making it very difficult to use for providing the power supply also of very low-power devices such as sensors. To overcome this limit, the electromagnetic field for remote powering applications can be generated ad hoc.

The remote powering exploiting RF signals (e.g., Industrial Scientific and Medical (ISM) band [1,2]) is very attractive from the application point of view, as the radiated electromagnetic field can reach locations (in the environment, on industrial machineries, in industrial plants), where no other kind of power sources is available and cables are difficult to be placed. In this respect, the remote wireless powering is exploited, for example, in bionic systems [3] and to power ultra-low-power devices like wireless sensor nodes [4,5]. The remote powering system is generally comprised of an antenna, an RF-DC power converter, and a power management circuit. The rectification circuits used to convert the RF power into a DC voltage is the most challenging building block of the whole system, whose optimization in terms of both conversion efficiency and sensitivity requires one to devise innovative solutions reducing the minimum power threshold needed for circuit operations.

Typically, in order to maximize the RF power gathered by the antenna, resonant matching networks are used to couple efficiently the Integrated Circuit (IC) converter with the antenna. Unfortunately, matching networks limit the frequency of operations around the resonance frequency, which is a problem in the case the signal/carrier frequency is not known a priori. Extending the frequency band of operations is thus highly desirable, as well as devising circuit solutions compensating the frequency shifts due to the unpredictable parasitic effects arising from the connection of the antenna to the converter IC. In this respect, the broadband co-design of the antenna and the IC converter is a key step to improve the efficiency and sensitivity performances of the whole system.

In this scenario, we present the design of an RF-DC energy conversion system comprised of the antenna and the IC converter. A reconfigurable differential RF-DC power converter architecture is selected to improve both conversion efficiency and sensitivity [6]. The converter is combined with a matched broadband PCB antenna [7] to enable the use of the RF energy conversion system at different frequencies in the ISM band. In order to minimize the power losses, no matching networks are used, and the antenna is designed to have an impedance matching the highly capacitive input of the IC converter.

Several papers presented in the literature focus on just one component (i.e., antenna or RF-DC converter) of an RF energy harvester. Differently from these contributions, this paper is focused on the presentation of a possible solution of a complete RF-DC energy harvesting system.

In particular, the issues related to the efficient combining of different system blocks are presented. Moreover, the whole system has been completely characterized by measurements carried out on the realized 
prototypes. The obtained results concerns the performance of the whole system, which is one of the most important things from an application point of view. Usually, the system architecture has to be chosen as a trade-off between opposite requirements of the single functional blocks, and combining the best solutions of each stand-alone component could not lead to the best performances of the whole system. For these reasons, we focused on the characterization of the whole system, in order to present some considerations and guidelines which can be used in the design of completely new RF energy harvesting systems.

The paper is organized as follows: The whole system is described in Section 2. The characterization results of the integrated circuit implementing the RF-DC converter and the smart voltage regulator are described in Section 3. In Section 4, we described the performances of the whole system. Conclusions are given in Section 5.

\section{System Description}

Figure 1 shows the RF energy harvesting system that we developed. It is comprised of (i) a custom differential antenna that gathers the incident RF energy; (ii) an RF-DC converter exploiting a reconfigurable architecture, which converts the RF power into a quasi-constant voltage supply; (iii) an output voltage regulator providing a constant DC voltage supply to an electrical load (e.g., an ULP wireless sensor node); (iv) an external logic circuit controlling the reconfigurable RF-DC converter.

Compared to classical solutions [6] the use of a reconfigurable architecture for the RF-DC converter allows for improving both efficiency and sensitivity of the whole system. At the same time, the use of a differential antenna designed to match the input impedance of the rectifier simplifies the overall system design, while improving the overall efficiency, as the power losses associated with the parasitics of the matching network that would be needed are intrinsically avoided.

\subsection{Reconfigurable Differential RF-DC Converter}

The reconfigurable RF-DC converter represents the core of the whole RF-DC energy harvesting system. It is comprised of two differential rectifier blocks derived from the classical two-stage Dickson voltage rectifier/multiplier [8] shown in Figure 2.

Each stage of the rectifiers is comprised of a clamp and rectifying sections: the clamp section adds a DC offset to the sinusoidal input voltage $V_{V R}$, while the rectifying section clamps the voltage to its maximum value. The steady state output voltage $V_{O U T}{ }_{-}$Stage depends on the input voltage amplitude, $V_{V R}$, and the voltage drop, $V_{M}$, across the transistors in diode configurations. Under the reasonable assumptions of identical transistors and negligible voltage drop across the large input capacitance, VOUT_Stage can be calculated as

$$
V_{\text {OUT_Stage }}=2\left(V_{V R}-V_{M}\right)
$$

It is evident that maximizing the efficiency of the voltage rectifier requires to minimize $V_{M}$, which requires adopting Metal-Oxide-Semiconductor Field-Effect Transistors (MOSFETs) with minimum threshold voltage, reverse current and on-resistance. This technology requirement is alleviated by the differential rectifier topology, which, thanks to the cross-connection scheme shown in Figure 2, allows for doubling the gate-source voltage of the MOSFETs compared to the classic diode-connected solutions. This is beneficial for the system efficiency, because it reduces the detrimental effects due to 
the threshold voltage of the MOSFETs. A detailed description of the operations of this proposed connection scheme is provided in [6].

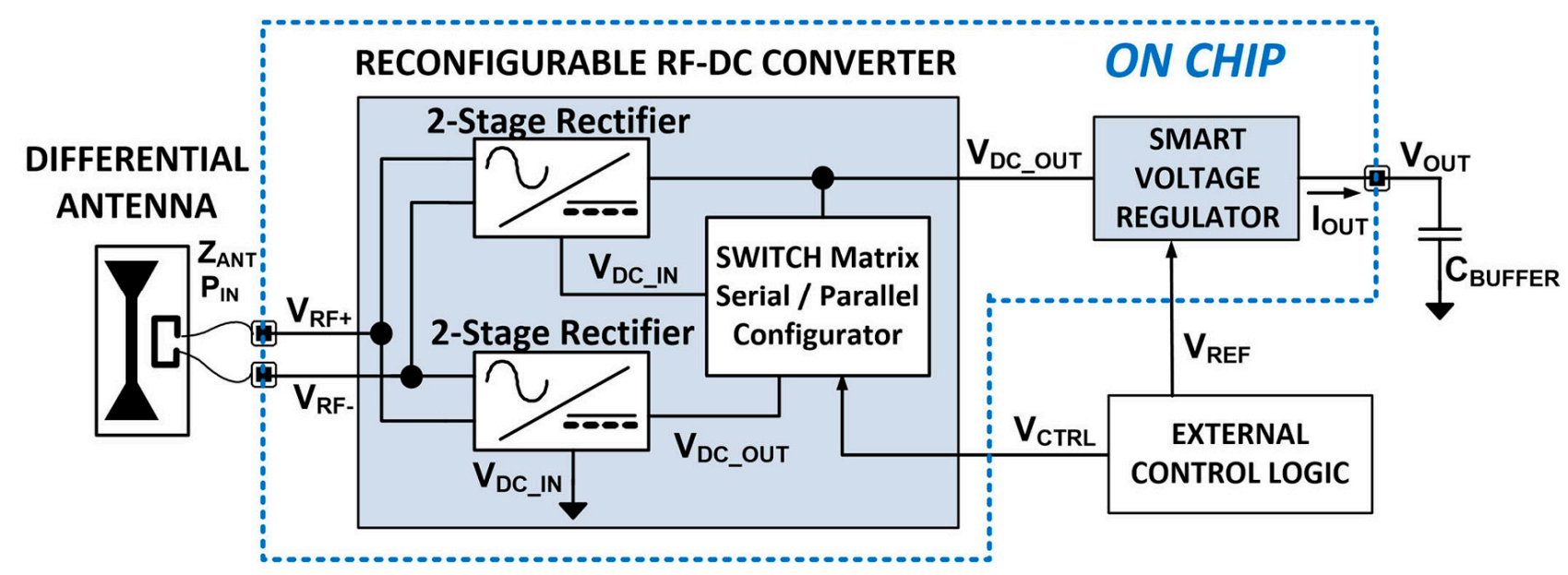

Figure 1. RF energy harvester system comprised of the custom differential antenna, the RF-DC converter, the voltage regulator, the external control logic controlling the converter reconfiguration, and the external capacitor $C_{B U F F E R}$ used for energy storage.
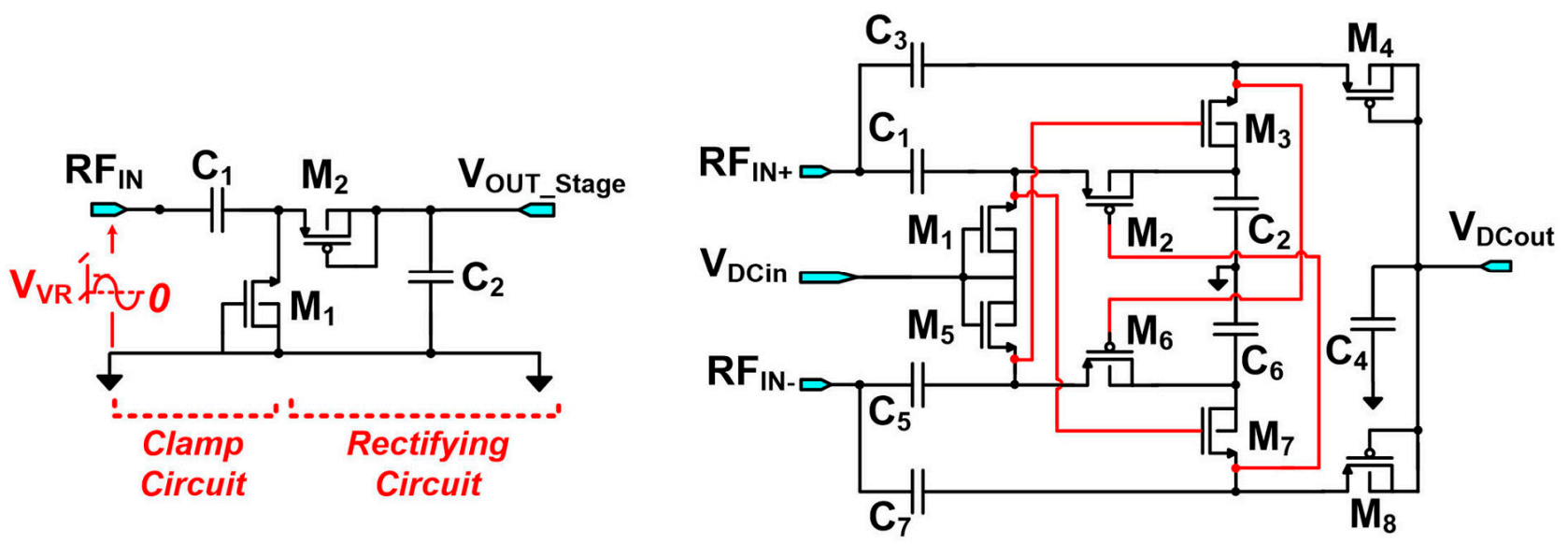

Figure 2. Basic cell of a classic Dickson voltage rectifier/multiplier (left) and the proposed two-stage reconfigurable differential rectifier block with cross connection scheme highlighted (right).

As shown in Figure 1, the two rectifier blocks can be connected either in series or in parallel by means of a CMOS Switch Matrix controlled by the External Control Logic accordingly to the available input RF power. Changing the connection between the two rectifier blocks allows modifying the number of stages of the whole RF-DC converter: a two-stage (four-stage) converter corresponds to the case of a parallel (series) connection. In turn, this allows for modifying the resistive part of the input impedance of the converter, $Z_{D}$, modeled by the parallel of $R_{D}$ and $C_{D}$, as shown in Figure 3, where $C_{D}$ accounts for the MOSFETs parasitic capacitance, while $R_{D}$ depends on the load and the active power flowing into the rectification circuit. Since $R_{D}$ depends also on the RF input power $P_{I N}$ [6], changing the rectifier connection allows for modifying $R_{D}$ to match the resistance of the antenna impedance $Z_{A N T}=R_{A N T}+j X_{A N T}$ at different $P_{I N}$ level. This allows for reducing the mismatch power losses due to the power-dependent 
variation of the input RF-DC converter, thus improving the efficiency of the matching network over a wider $P_{I N}$ range. The impedance matching between the antenna and the converter is designed to account for the parasitic capacitance of the pad $C_{P A D}$, and the ohmic power losses due to the bonding parasitic resistance, $R_{B o n d}$, and inductance $L_{B o n d}$, which are included into the input impedance model, as shown in Figure 3, [9]. Furthermore, the reconfigurable circuit solution devised for the RF-DC converter allows for minimizing the efficiency reduction due to the $R_{D}$ variation caused by the voltage regulator operation.

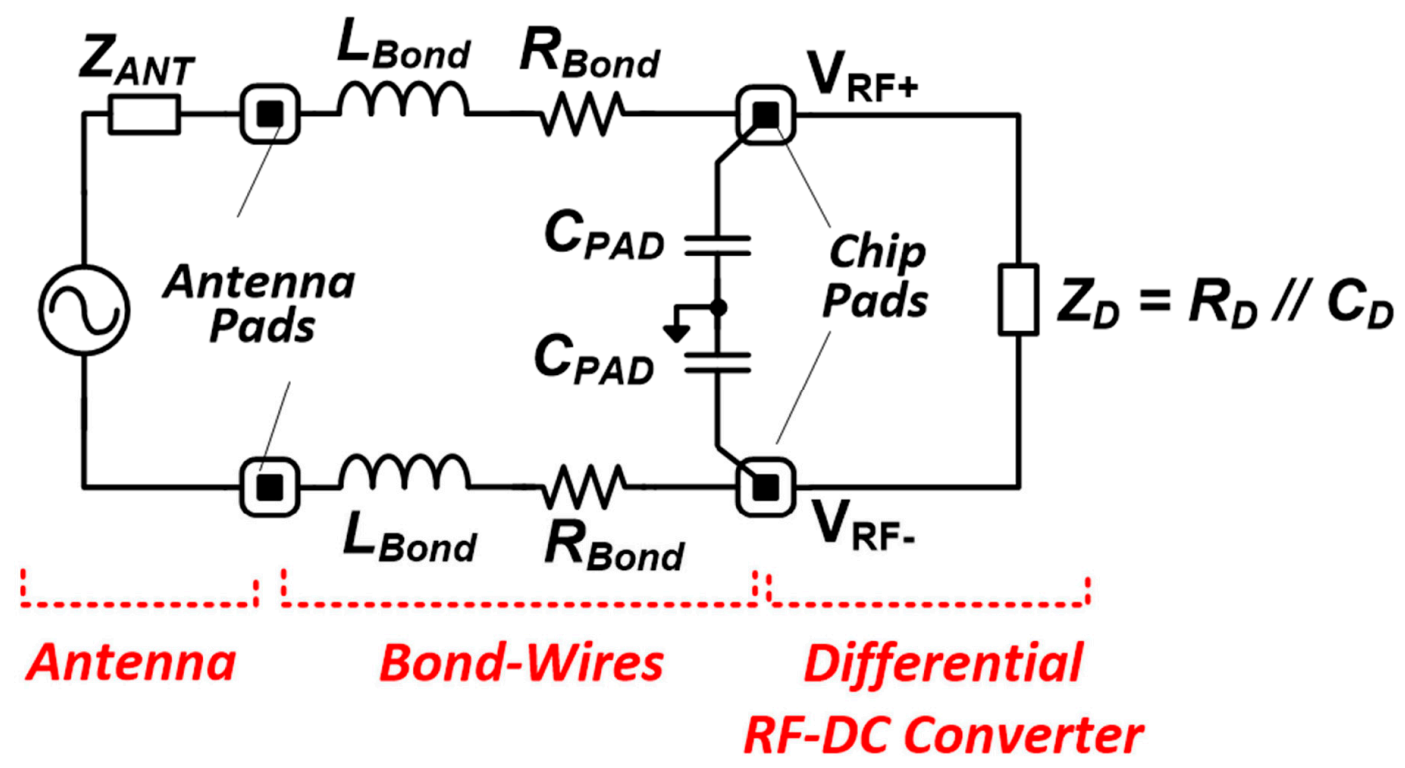

Figure 3. Equivalent circuit used for the design of the impedance matching between the antenna and the differential RF-DC converter. $Z_{A N T}$ is the antenna impedance; $L_{B o n d}$ and $R_{B o n d}$ are the parasitic resistance and inductance of the two bondwires, respectively; $C_{P A D}$ is the pad capacitance; $Z_{D}$ is the input impedance of the differential RF-DC converter within the ISM band modeled as the parallel of the the equivalent resistance $R_{D}$ and the equivalent capacitance $C_{D}$.

\subsection{Smart Output Voltage Regulator}

The Smart Voltage Regulator (SVR) has been designed to keep the converter output voltage, VOUT, at the constant reference voltage $V_{R E F}=2 \mathrm{~V}$. With reference to Figure 1, $V_{R E F}$ is set by the external control logic.

The operating principle of the SVR circuit, which resembles a classical current mirror, is very simple. With reference to Figure 4, the transistors $M_{A}$ and $M_{C}$ keep constant the gate voltage of the transistor $M_{B}$. When the available antenna input power $P_{I N}$ increases (decreases), VOUT is forced to rise (fall). The higher (lower) VOUT determines an increase (decrease) of the source-gate voltage of $M_{B}$, hence also the current drained by $M_{B}$ increases (decreases). The lower (higher) equivalent DC resistance at the output of the RF-DC Converter forces the decrease (increase) of VOUT, counteracting the initial VOUT variation. Therefore, the SVR behaves as an active load.

Besides keeping the output voltage constant, the SVR significantly benefits the overall circuit efficiency compared to solutions employing either voltage limiters or LDO regulators. In fact, with the proposed solution, the output current, IOUT, charging the external buffer capacitor, $C_{B U F F E R}$, is modulated according to the available input power $P_{I N}$. This allows for storing the excess input power into $C_{B U F F E R \text {, while }}$ 
keeping constant the power dissipated across $M_{B}$. Thus, the SVR efficiency remains constant regardless the operating conditions.

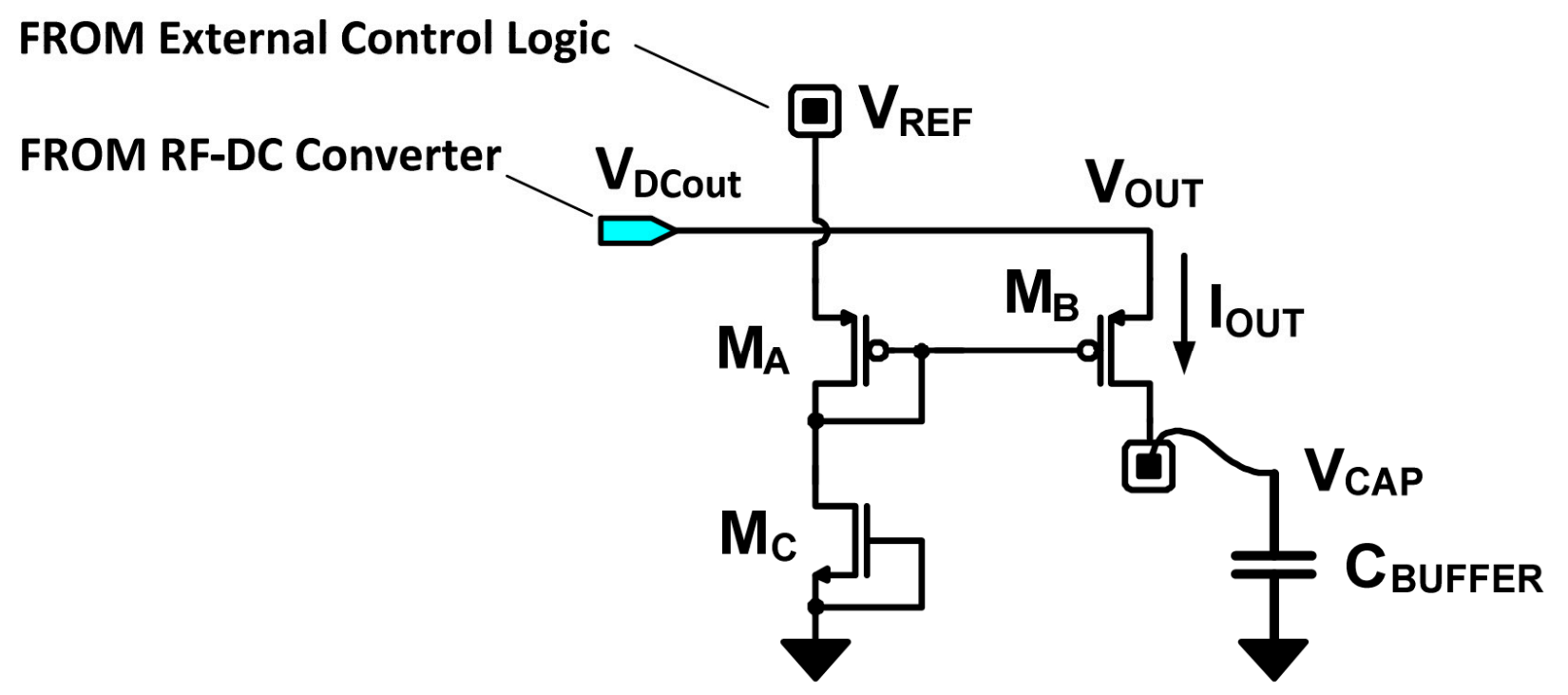

Figure 4. Transistor level implementation of the Smart Voltage Regulator.

\subsection{Custom Differential Antenna}

The custom differential antenna has been realized on a low cost FR4 substrate and exploits a layout reproducing a printed bi-conical radiating dipole with linear polarization. A rectangular loop couples inductively the dipole with the chip, as shown in Figure 5. This topology has been chosen because it allows one to adjust independently the real and the imaginary parts of the antenna impedance acting on the size of the loop and its distance from radiating dipole [10].

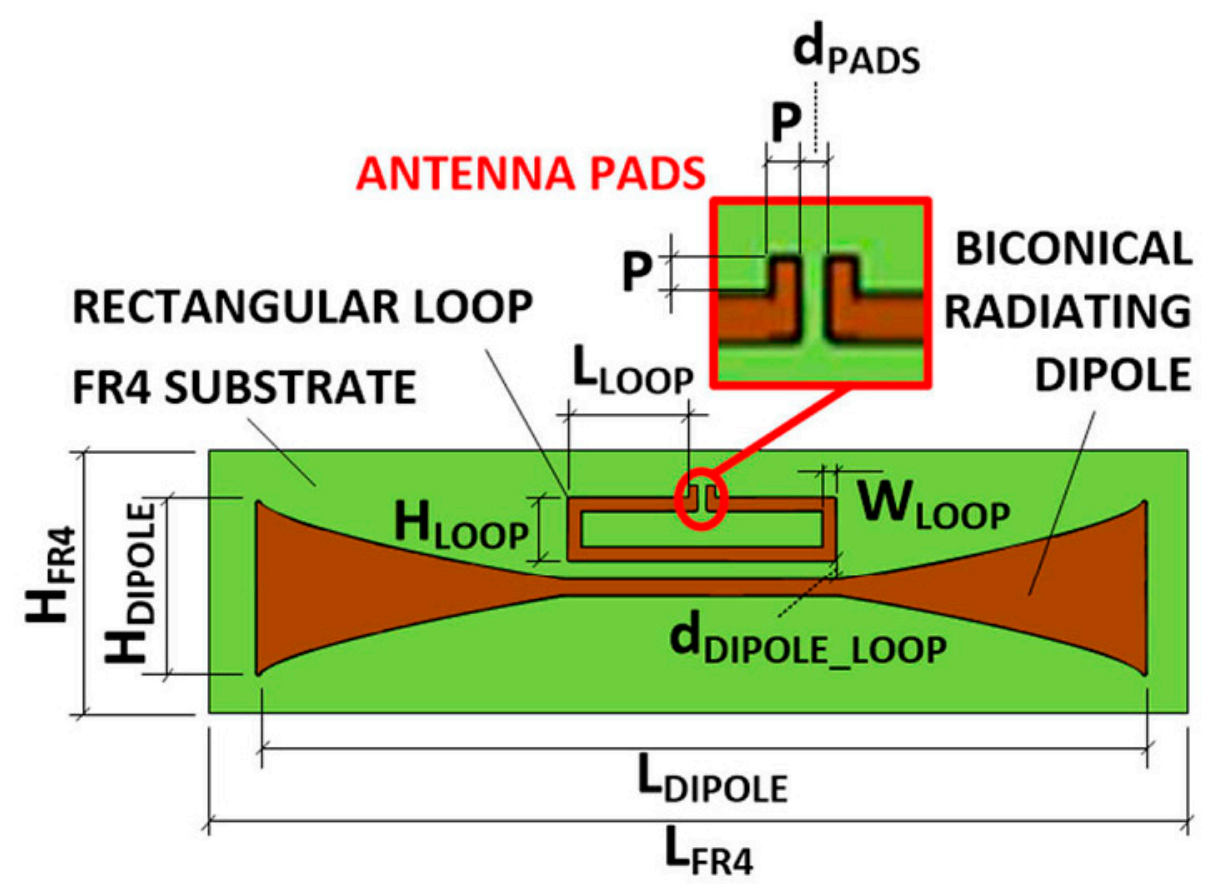

Figure 5. Cont. 


\begin{tabular}{|c|c|c|l|}
\cline { 2 - 4 } \multicolumn{1}{c|}{} & PARAMETER & VALUE $(\mathrm{mm})$ & \multicolumn{1}{c|}{ DESCRIPTION } \\
\hline \multirow{3}{*}{$\begin{array}{c}\text { BICONICAL } \\
\text { DIPOLE }\end{array}$} & $\mathrm{L}_{\text {DIPOLE }}$ & 101 & dipole length \\
\cline { 2 - 4 } & $\mathrm{H}_{\text {DIPOLE }}$ & 20 & dipole height \\
\hline \multirow{4}{*}{$\begin{array}{c}\text { RECTANGULAR } \\
\text { LOOP }\end{array}$} & $\mathrm{L}_{\text {LOOP }}$ & 14.7 & half loop length \\
\cline { 2 - 4 } & $\mathrm{H}_{\text {LOOP }}$ & 10 & loop height \\
\cline { 2 - 4 } & $\mathrm{W}_{\text {LOOP }}$ & 1 & loop width \\
\cline { 2 - 4 } & $\mathrm{d}_{\text {DIPOLE_LOP }}$ & 2 & distance dipole-loop \\
\hline \multirow{3}{*}{ ANTENNA PADS } & $\mathrm{P}$ & 1 & pad size \\
\cline { 2 - 4 } & $\mathrm{d}_{\mathrm{PADS}}$ & 0.6 & distance between pads \\
\hline & $\mathrm{L}_{\mathrm{FR} 4}$ & 110 & substrate length \\
\cline { 2 - 4 } & $\mathrm{H}_{\mathrm{FR} 4}$ & 30 & substrate height \\
\cline { 2 - 4 } & $\mathrm{t}_{\mathrm{TOT}}$ & 0.8 & FR4 total thickness \\
\cline { 2 - 4 } & $\mathrm{t}_{\mathrm{CU}}$ & 0.035 & Cu thickness \\
\hline
\end{tabular}

Figure 5. Layout of the differential antenna realized on a FR4 substrate (up) and geometrical parameters of the antenna (down).

This significantly simplifies the design procedure, which requires one to match the antenna resistance and reactance to the converter impedance in order to maximize the power transfer and conversion. To this purpose, we also included the pad parasitic capacitance $C_{P A D}=200 \mathrm{fF}$, and the impedance of the bond-wires used to connect the antenna to the IC, which have a resistance and an inductance of $R_{\text {Bond }}=2 \Omega$ and $L_{\text {Bond }}$ $=1 \mathrm{nH}$, respectively.

For the antenna design, we used the electromagnetic simulations performed with the CST Microwave Studio Software, which allowed including the effect of the antenna substrate, i.e., FR4 with relative dielectric permittivity $\varepsilon_{r}=4.3$ and thickness of $0.8 \mathrm{~mm}$. The antenna was designed by assuming an input impedance of the voltage regulator with $R_{D}=10 \mathrm{k} \Omega$ and $C_{D}=400 \mathrm{fF}$, with a pad and bonding parasitics equal to $C_{P A D}=200 \mathrm{fF}, R_{B o n d}=2 \mathrm{~W}$, and $L_{B o n d}=1 \mathrm{nH}$, respectively.

Unfortunately, the impedance matching can be effective only around the resonance frequencies, and, therefore, the design of a broadband antenna results in a very challenging task. In order to widen the band of the antenna, we designed the antenna so that its imaginary part matches the rectifier input capacitance at $900 \mathrm{MHz}$ whereas the real one is at $840 \mathrm{MHz}$ [11]. In addition, the bi-conical shape of the radiating element allows further increasing the bandwidth. Simulation results, compared with the measured ones, are shown in Figure 6.

The slight penalty paid in terms of power transfer is widely compensated by the larger bandwidth as shown in Figure 7 where the power transfer coefficient $\tau$, [12]

$$
\tau=4 \frac{R_{D} R_{a n t}}{\left|Z_{D}+Z_{\text {ant }}\right|^{2}}
$$

is reported. The power transfer coefficient never reaches the maximum value of 1 , corresponding to the perfect matching condition; however, it is higher than 0.5 , corresponding to a $3 \mathrm{~dB}$ of loss, over a range of $130 \mathrm{MHz}$. 

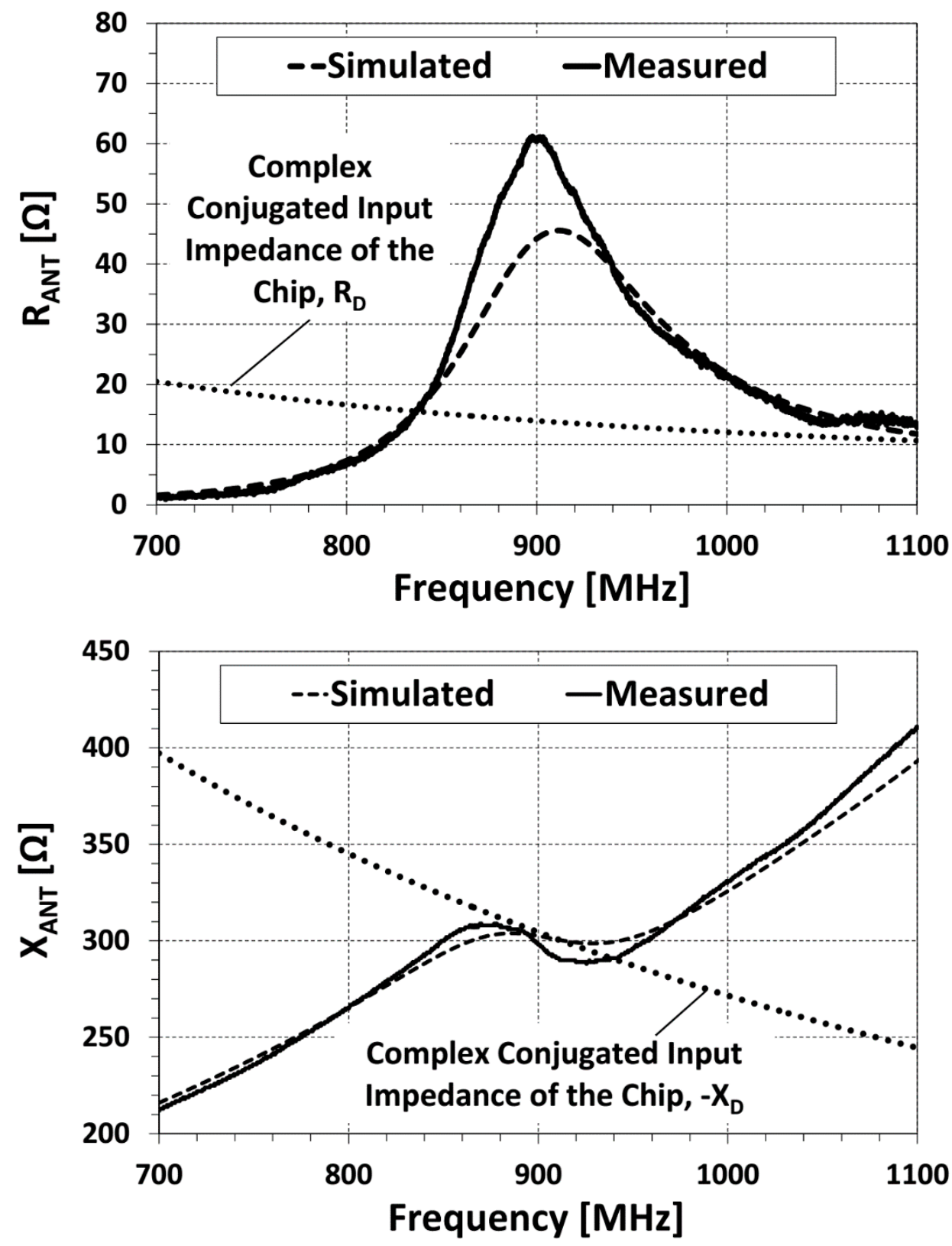

Figure 6. The real (up) and imaginary (down) parts of the measured (solid line), simulated (dashed line) input antenna impedance, and of the complex conjugated input impedance RF-DC converter chip (dotted line).

Figure 8 (left) shows the antenna prototype we realized. The measurement of the input impedance is a quite critical step due to the symmetrical input port, which should require a balun to connect it with the coaxial cable of the network analyzer, and due to the high mismatch of the antenna impedance with standard $50 \Omega$ components. One effective solution to mitigate these problems is the set-up shown in Figure 8 (right). Thanks to the antenna symmetry, we just used a half of the antenna placed on a $1 \mathrm{mx} 1 \mathrm{~m}$ copper ground plane [7]. The antenna was connected to the network analyzer through a coaxial cable placed on the other side of the copper plane. The measured input impedance and power transfer coefficient are compared with simulation results in Figures 6 and 7 showing a good agreement. The reduction of the experimental value of the power transfer coefficient at $900 \mathrm{MHz}$ is due to the higher prototype antenna resistance with respect to the numerical design. 


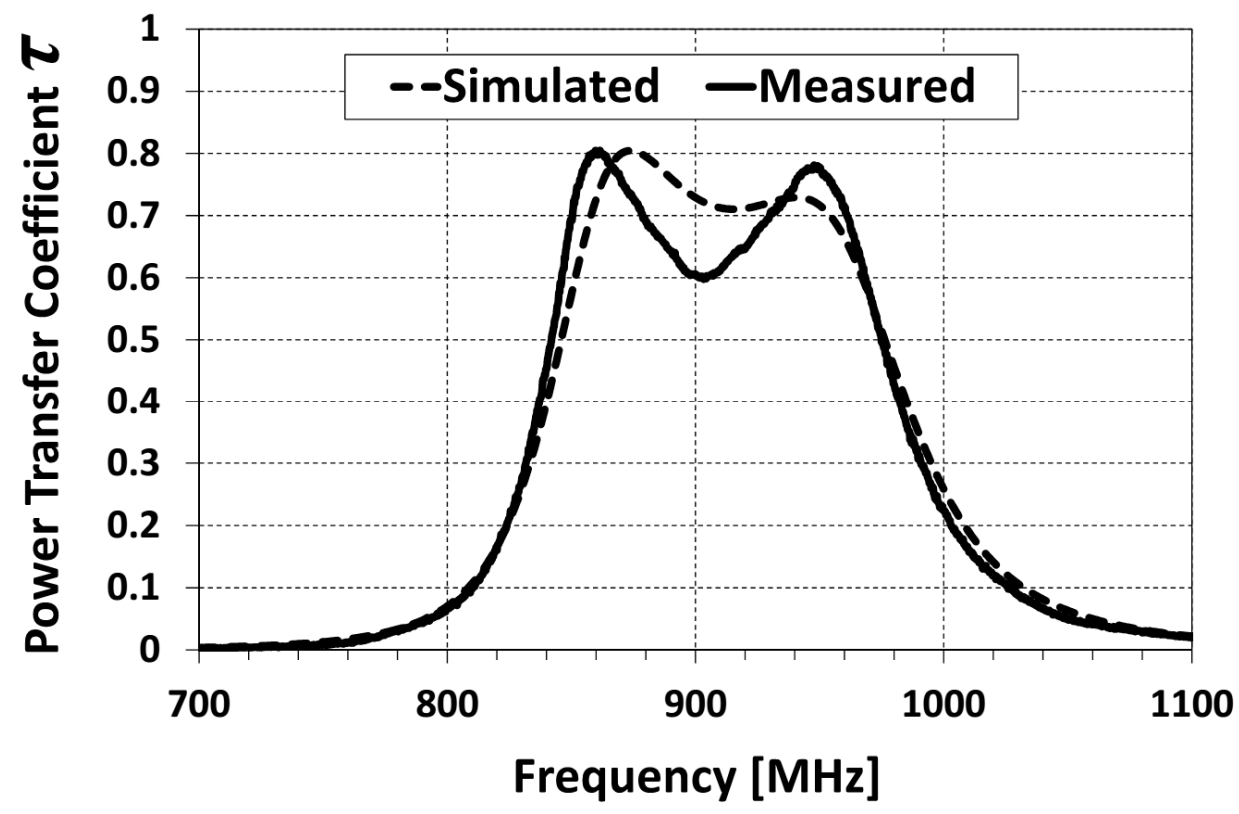

Figure 7. The measured (solid line) and simulated (dashed line) power transfer coefficient between antenna and chip.
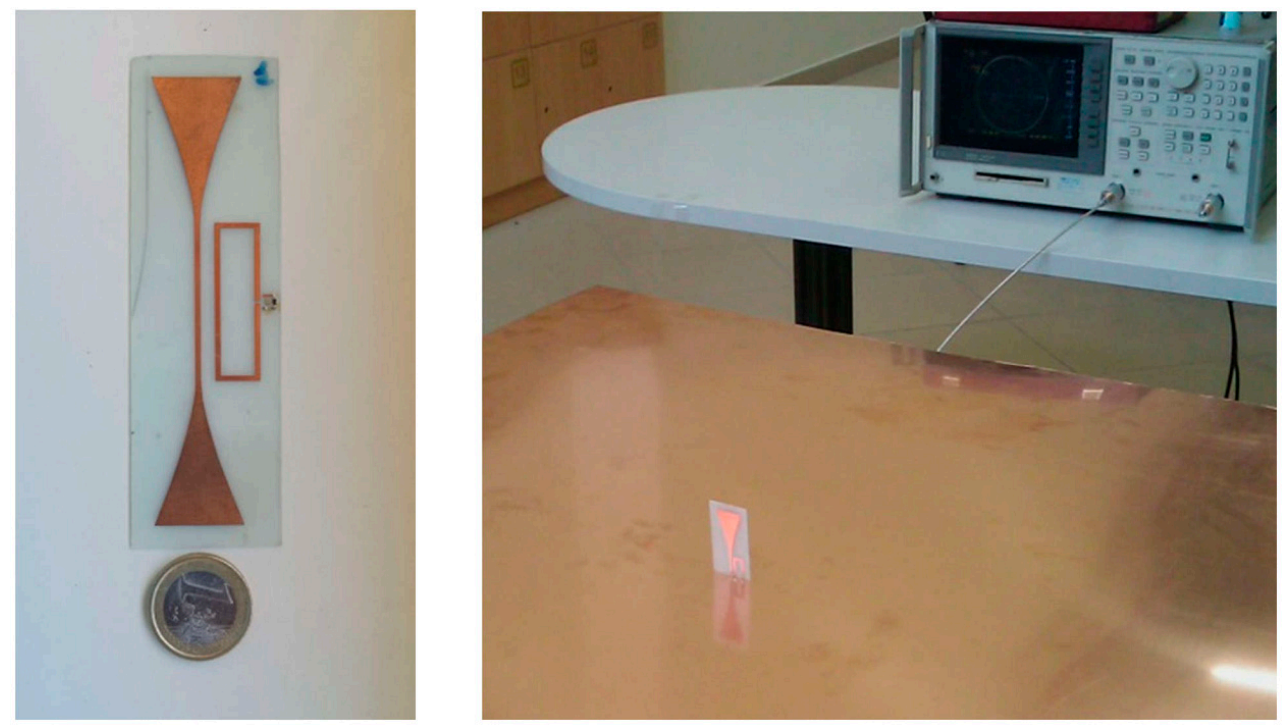

Figure 8. Antenna prototype (left) and antenna input impedance measurement set-up (right).

\section{Realization and Characterization Results of the IC Converter}

The integrated circuit implementing the RF-DC converter and the Smart Voltage Regulator has been realized in STM $130 \mathrm{um}$ technology. The chip area is of $550 \times 400 \mu \mathrm{m}^{2}$.

We characterized the performances of the RF-DC converter including the smart voltage regulator by measuring the output voltage and the converter efficiency. Such measurements have required an ad hoc measurement setup because of the following reasons: (i) the network analyzer and all the RF instrumentation is single-ended, thus mandating the adoption of a balun for the single-ended to differential conversion; (ii) the characteristic input impedance of the RF instruments, $50 \Omega$, requires one to be to matched the input impedance of the differential RF-DC converter for an accurate measurement. In order to overcome 
the above issues, we implemented a dedicated measurement setup for the characterization of the differential RF-DC converter, which is schematized in Figure 9. The setup is comprised of a Network Analyzer, which emulates a $50 \Omega$ single-ended antenna and allows for measuring the reflection coefficient $S_{11}$, the PCB Balun and the PCB testing board embedding both the converter and the matching network required to match the $50 \Omega$ output impedance of the Balun with the input impedance of the converter. A $1.5 \mathrm{~dB}$ insertion loss has been considered to account for interconnections and cables.

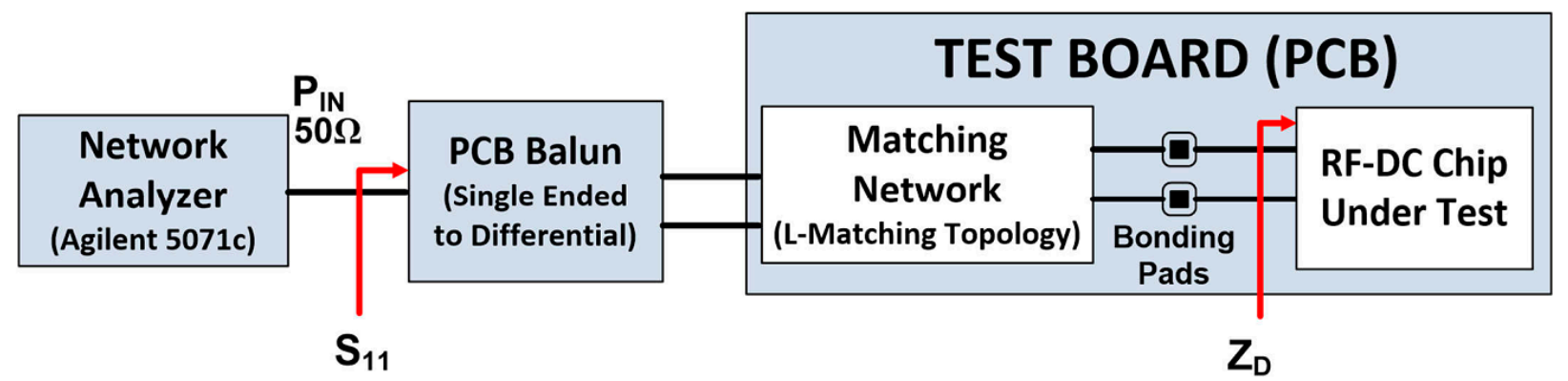

Figure 9. Measurement setup developed for the characterization of the differential RF-DC converter. It is comprised of a Network Analyzer, a PCB BalUn and a PCB test board embedding the Matching Network and the chip under test. Bonding parasitics have been taken into account in the design of the Matching Network.

Figure 10 shows the output voltage measured at different input power level, which is around the reference voltage of $\sim 2 \mathrm{~V}$ for the whole $\sim 20 \mathrm{~dB} P_{I N}$ range. The converter starts working at $-17 \mathrm{dBm}$, demonstrating a very high sensitivity. The IC efficiency calculated over the whole $P_{I N}$ range peaks at $60 \%$, while remaining above $40 \%$ over a $14 \mathrm{~dB}$ input power range. The above performances, among the highest ones reported in the literature, are adapted from [0] and prove the effectiveness of the reconfigurable architecture proposed for the differential converter.

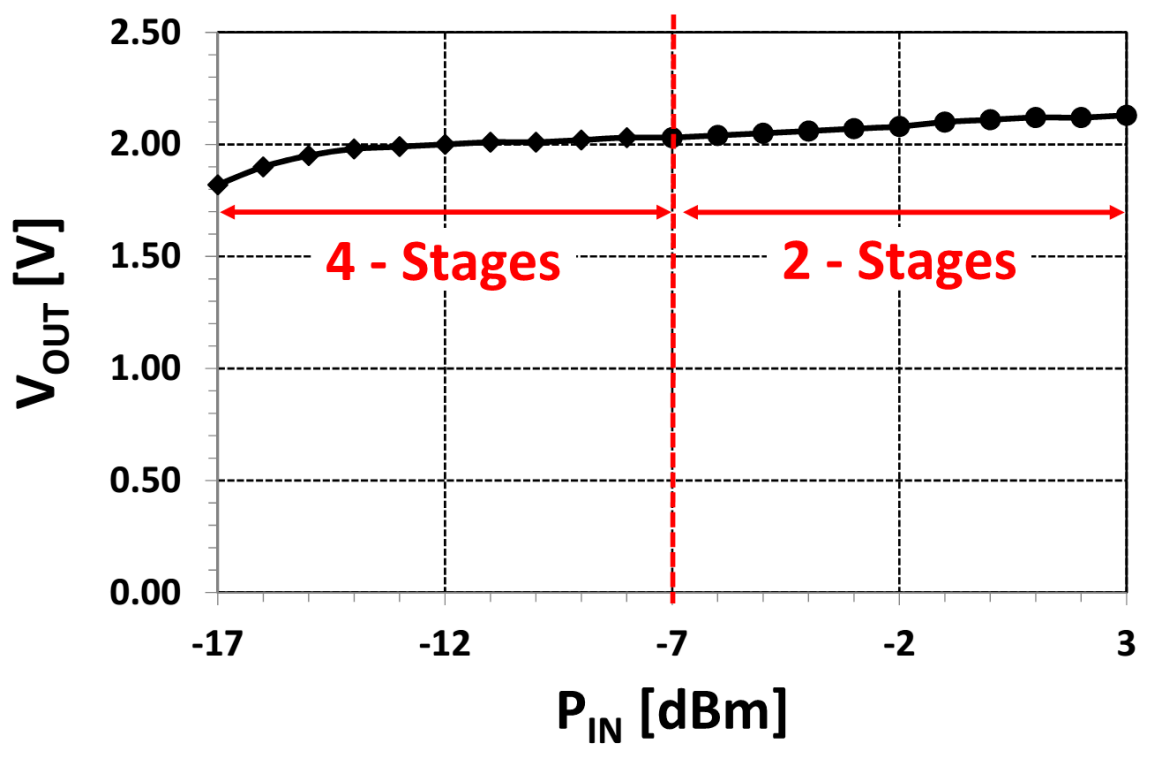

Figure 10. Cont. 


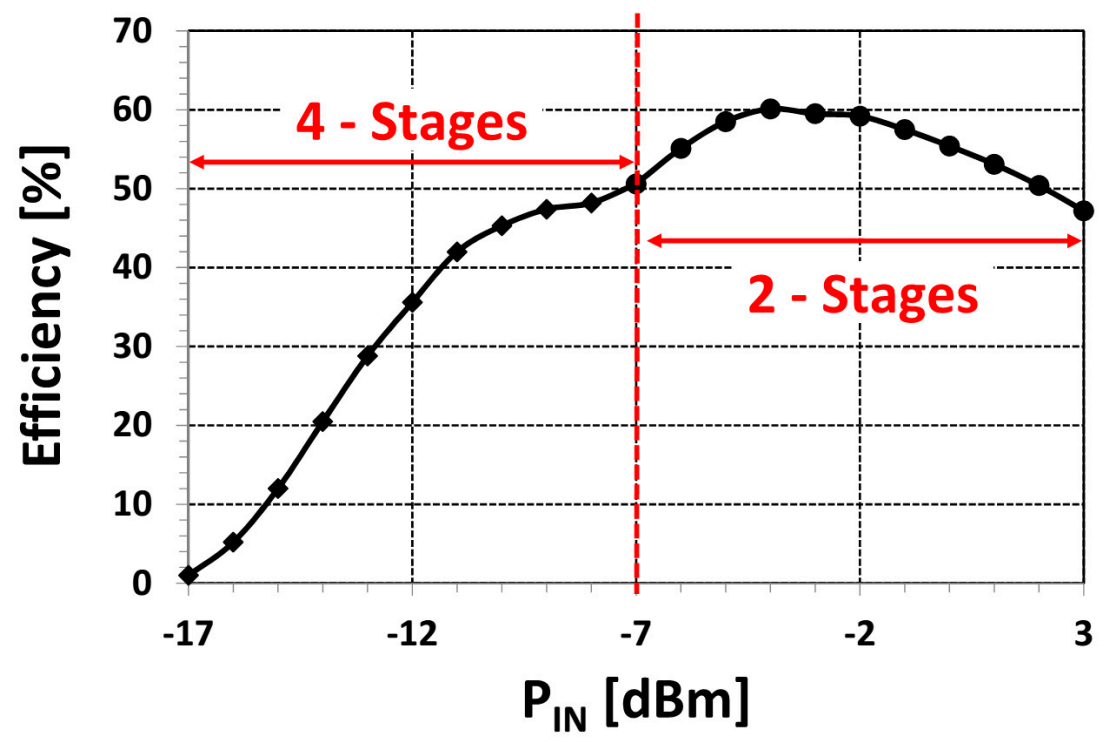

Figure 10. Output voltage (up) and converter efficiency (down) measured at different input power levels, $P_{I N}$, at $869 \mathrm{MHz}$. Similar results have been obtained at $915 \mathrm{MHz}$.

In order to properly design the antenna, we measured also the input impedance of the RF-DC converter. The differential resistance and capacitance measured at the voltage rectifier input are shown in Figure 11 for both two-stage and the four-stage configurations. The contribution of the Balun and the PCB matching network have been de-embedded to improve the measurement accuracy. Simulations show a good agreement with measurements, except for $R_{D}$ measured at input power level lower than $-10 \mathrm{dBm}$.
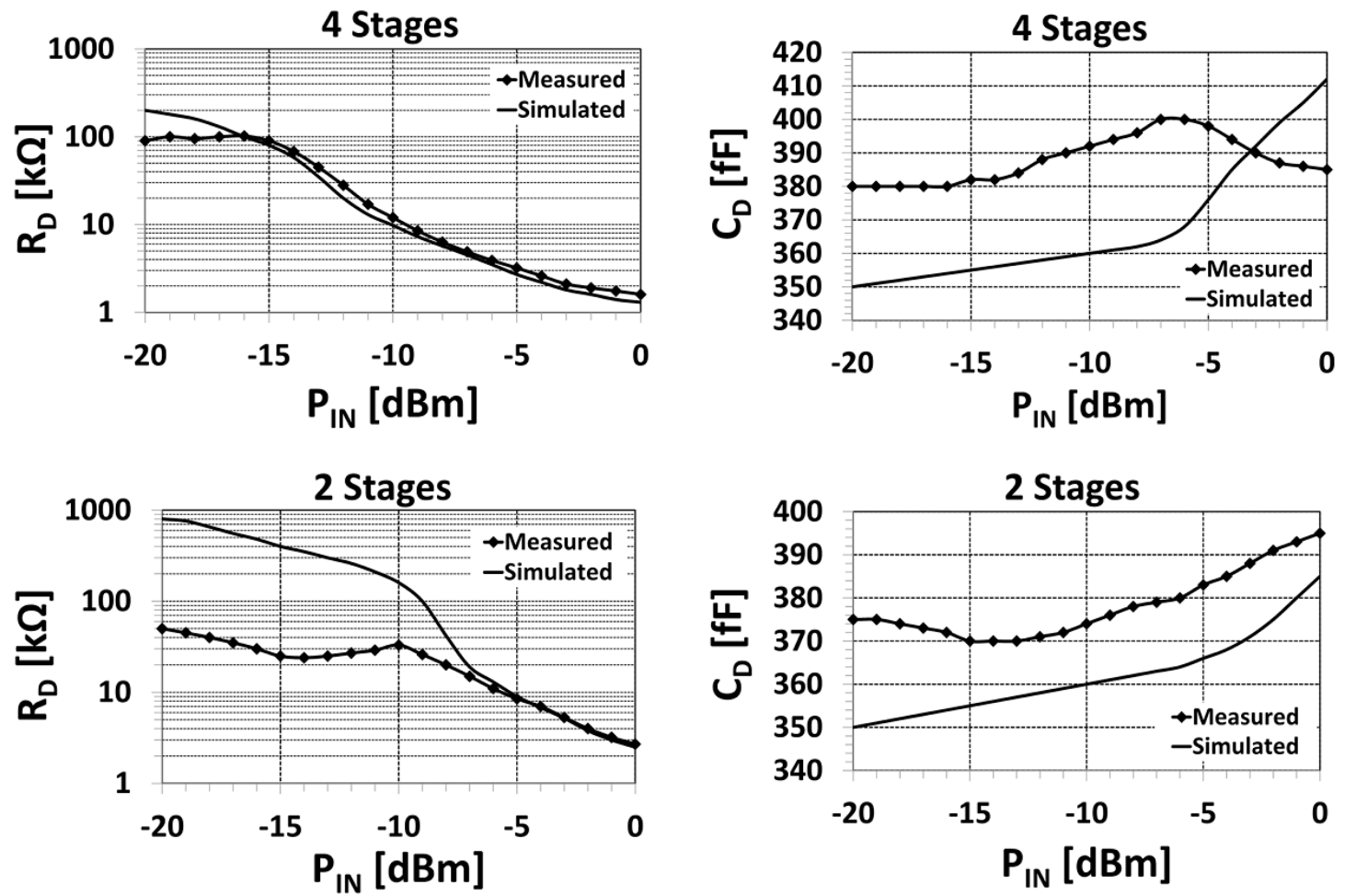

Figure 1 Comparison between simulated and measured input impedance (i.e., $\left.R_{D} / / C_{D}\right)$ of the differential voltage rectifier. The input impedance is compared both for the parallel (two-stage) and series (four-stage) connection of the RF-DC Converter basic blocks. 
The discrepancies of the simulated and measured input $R_{D}$ of the RF-DC converter are due to inaccuracy of the instrumentation for high values of $R_{D}$. The reason of this inaccuracy is due to the fact that a high $R_{D}$ determines a $\left|S_{11}\right| \mathrm{dB}$ measured with the Network Analyzer around $0 \mathrm{~dB}$, and, in this condition, the Network Analyzer is less accurate.

In more detail, the inaccuracies are mainly due to the complex measurement setup we have been forced to use (see Figure 9). From one side, all the available instrumentations are single ended and adapted to 50 Ohm. From the other side, the RF-DC converter is differential and its input impedance is not 50 Ohm and it varies accordingly with the available RF input power and the specific configuration (two-stage or four-stage). Consequently, in order to characterize the input impedance of the RF-DC converter measuring the $S_{11}$ parameter by means of a standard Network Analyzer, we had to insert a PCB balun for the single ended to differential adaption. Moreover, we also needed a tunable matching network between the balun and the RF-DC converter.

The above measurements of the rectifier input impedance were used for the design of the custom PCB antenna.

\section{Realization and Characterization Results of the Whole RF Harvester System}

The converter chip has been mounted on the PCB antenna realized on a FR4 substrate, while the external control logic has been implemented on a separate board.

The prototype of the whole RF energy harvester has been characterized using the experimental setup used illustrated in Figure 12, which was developed to emulate the standard working conditions occurring in real outdoor scenarios.

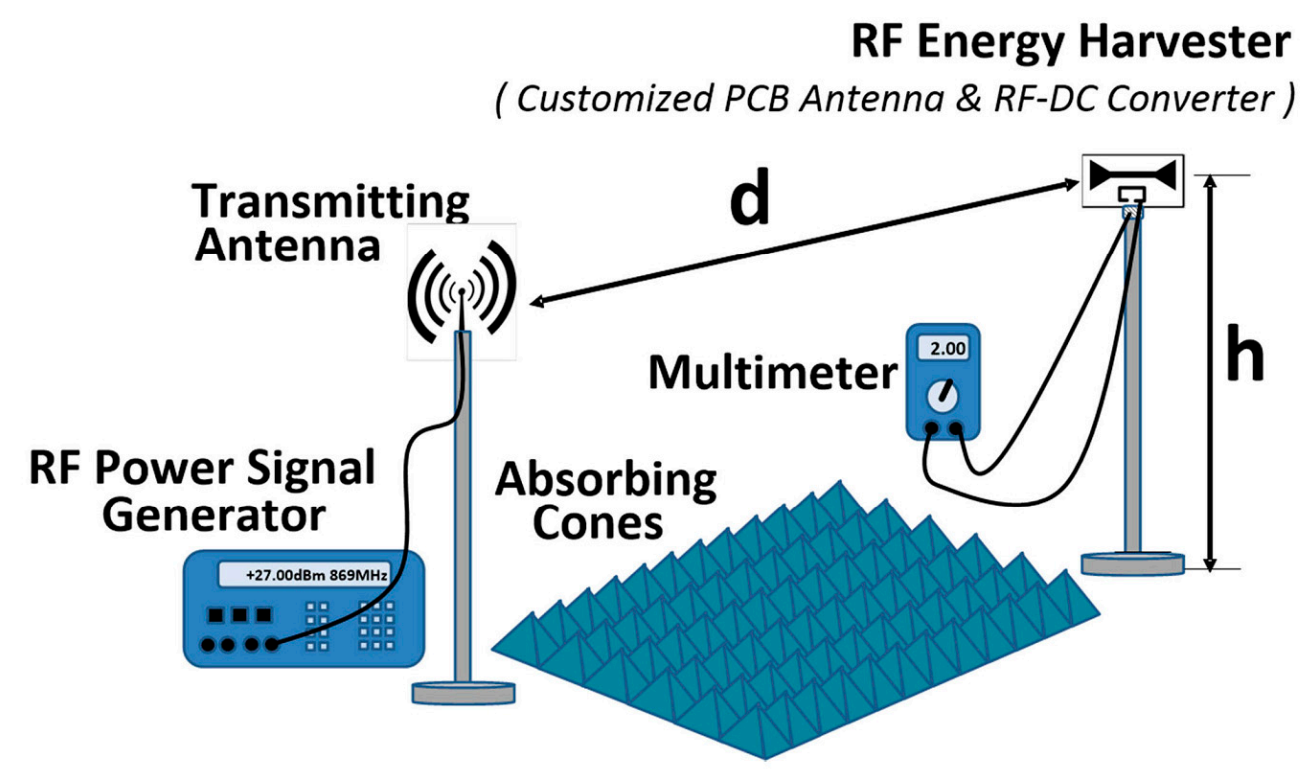

Figure 12. Setup used for the characterization of the whole system. Absorbing cones have been added to reduce the influence of reflected waves. The output current of the Smart Voltage Regulator has been measured by means of a digital multimeter in series.

The RF power signal, generated using a N5181A MXG signal generator from Agilent Technologies, is delivered to the RF energy harvesting module through a commercial broadband log-periodic transmitting 
antenna with linear polarization. The aligned antenna and RF energy harvester system were positioned in the maximum link direction at a distance $d=1.5 \mathrm{~m}$. Their height from the soil was $h=1.8 \mathrm{~m}$. A digital multimeter measured the output current of the harvester, i.e., the output of the Smart Voltage Regulator that maintains the output of the rectifier at $2 \mathrm{~V}$ modulating the rectifier output current. The losses due to the RF cables were de-embedded.

The characterization of the system was primarily focused on the evaluation of the energy harvesting efficiency, $\eta$, of the whole system

$$
\eta=\frac{P_{O U T}}{P_{I N}}
$$

where POUT is the power measured at the output of the RF energy harvester, $P_{\text {OUT }}=V_{\text {OUT }} \times I_{\text {OUUT }}$. VOUT is the rectifier output voltage; IOUT is the output current of the smart voltage regulator. $P_{I N}$ is the power available at the input of the chip in case of perfect antenna-chip matching condition. It can be estimated indirectly from the Friis's law [14]:

$$
P_{I N}=P_{T X} g_{T X}\left(\theta_{T X}, \varphi_{T X}\right) g_{R X}\left(\theta_{R X}, \varphi_{R X}\right) \chi\left(\frac{\lambda}{4 \pi d}\right)^{2}
$$

where $P_{T X}$ is the power transmitted by the RF signal generator; $g_{T X}, g_{R X}$ are the gain functions of the transmitting and receiving antennas along the direction $\theta, \phi$ respectively; $\chi$ the polarization loss factor; $\lambda$ is the wavelength of the transmitted RF signal; $d$ is the distance between the transmitting antenna and the RF harvester. In the set-up, the antenna was polarization matched and aligned for maximum directional radiation and reception, thus Equation (3) reduces to:

$$
P_{I N}=P_{T X} G_{T X} G_{R X}\left(\frac{\lambda}{4 \pi d}\right)^{2}
$$

being $G_{T X}, G_{R X}$ the gain of the transmitting and receiving antennas, respectively, and they were separately characterized. For example, at the two frequencies compliant with regulations in force in North America and Europe (i.e., $915 \mathrm{MHz}$ and $869 \mathrm{MHz}$, respectively), the obtained gains have been $G_{T X}=7.9(8.2) \mathrm{dBi}$ at $915(869) \mathrm{MHz} ; G_{R X}=1.6(1.4) \mathrm{dBi}$ at $915(869) \mathrm{MHz}$.

Figure 13 shows the efficiency of the whole system measured varying both the frequency and the power of the RF wave incident over the energy harvester. Efficiency curves obtained at $915 \mathrm{MHz}$ and $869 \mathrm{MHz}$ have been highlighted. When the series connection is selected (i.e., four-stage configuration), the voltage rectifier shows the best sensitivity, supplying a regulated $2 \mathrm{~V}$ output voltage starting from $P_{I N}=-12.5 \mathrm{dBm}$ (at $915 \mathrm{MHz}$ ). On the other hand, the parallel connection for the rectifier (i.e., 2-stages configuration) shows the highest conversion efficiency, but it needs higher levels of $P_{I N}$ to outperform the performances of the series configuration.

Figure 14 shows the system maximum efficiency plotted $v s$. the frequency for both the series connection (i.e., four-stage) and the parallel connection (i.e., two-stage). Noticeably, the proposed system demonstrates an efficiency higher than $15 \%$ over a $70 \mathrm{MHz}(55 \mathrm{MHz})$ wide-band centered at $915 \mathrm{MHz}$ in case of parallel (series) connection, differing from typically narrowband RF energy harvester solutions. This is due to the implemented broadband antenna which has been designed to match the imaginary part of the chip input impedance at $900 \mathrm{MHz}$, while the real part of the antenna impedance has been chosen 
higher than the real part of the chip input impedance. This choice, as confirmed by the measurements, from one side allows to broaden the bandwidth as desired, on the other side does not allow to obtain the maximum power transfer at $900 \mathrm{MHz}$.

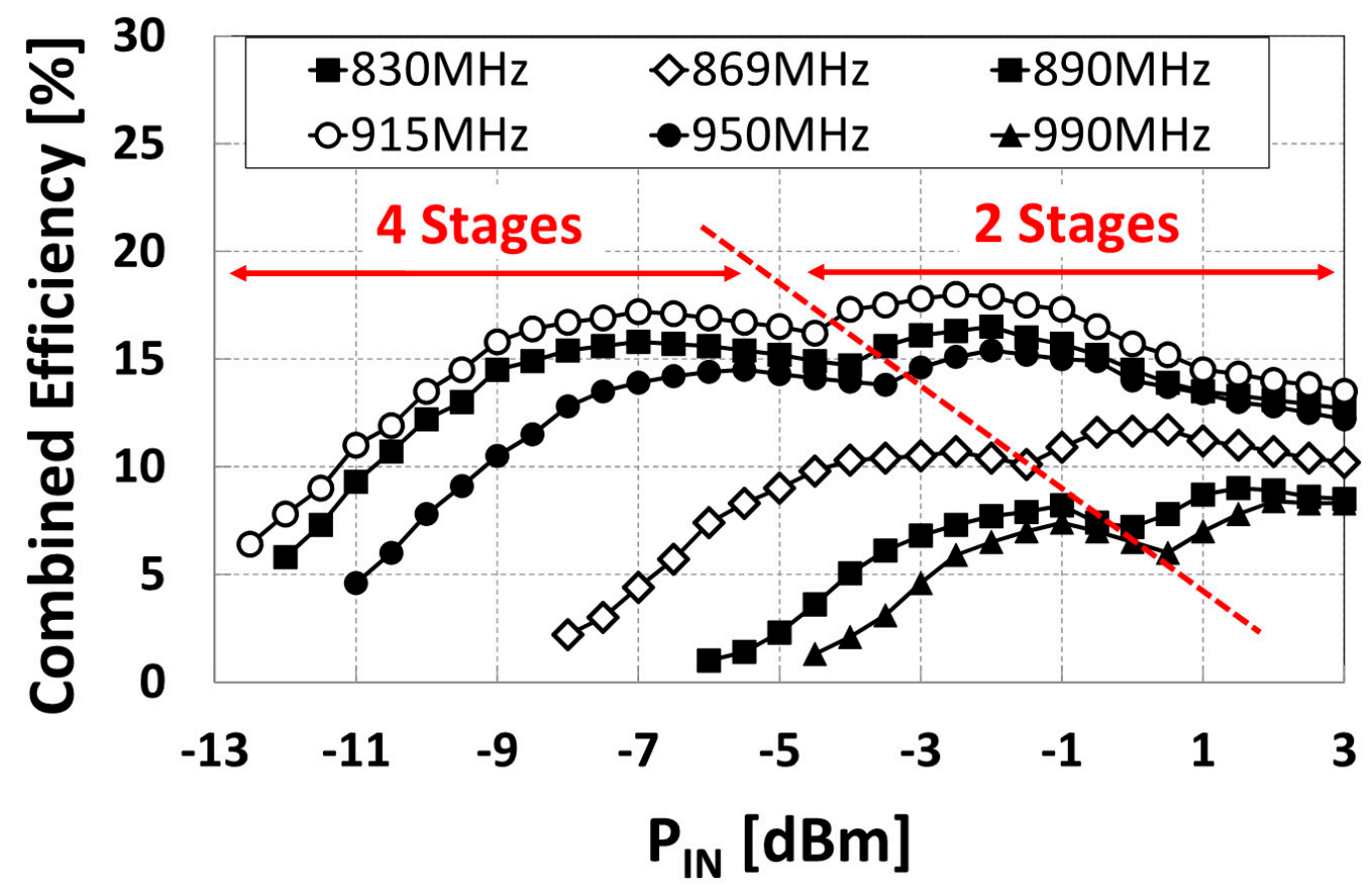

Figure 13. Efficiency of the whole RF energy harvesting system $v s$. the RF input power PIN for different frequencies (not all the measured curves are shown for the sake of clarity of the figure). The frequencies compliant with regulation in force in Europe and North America are $869 \mathrm{MHz}$ and $915 \mathrm{MHz}$, respectively.

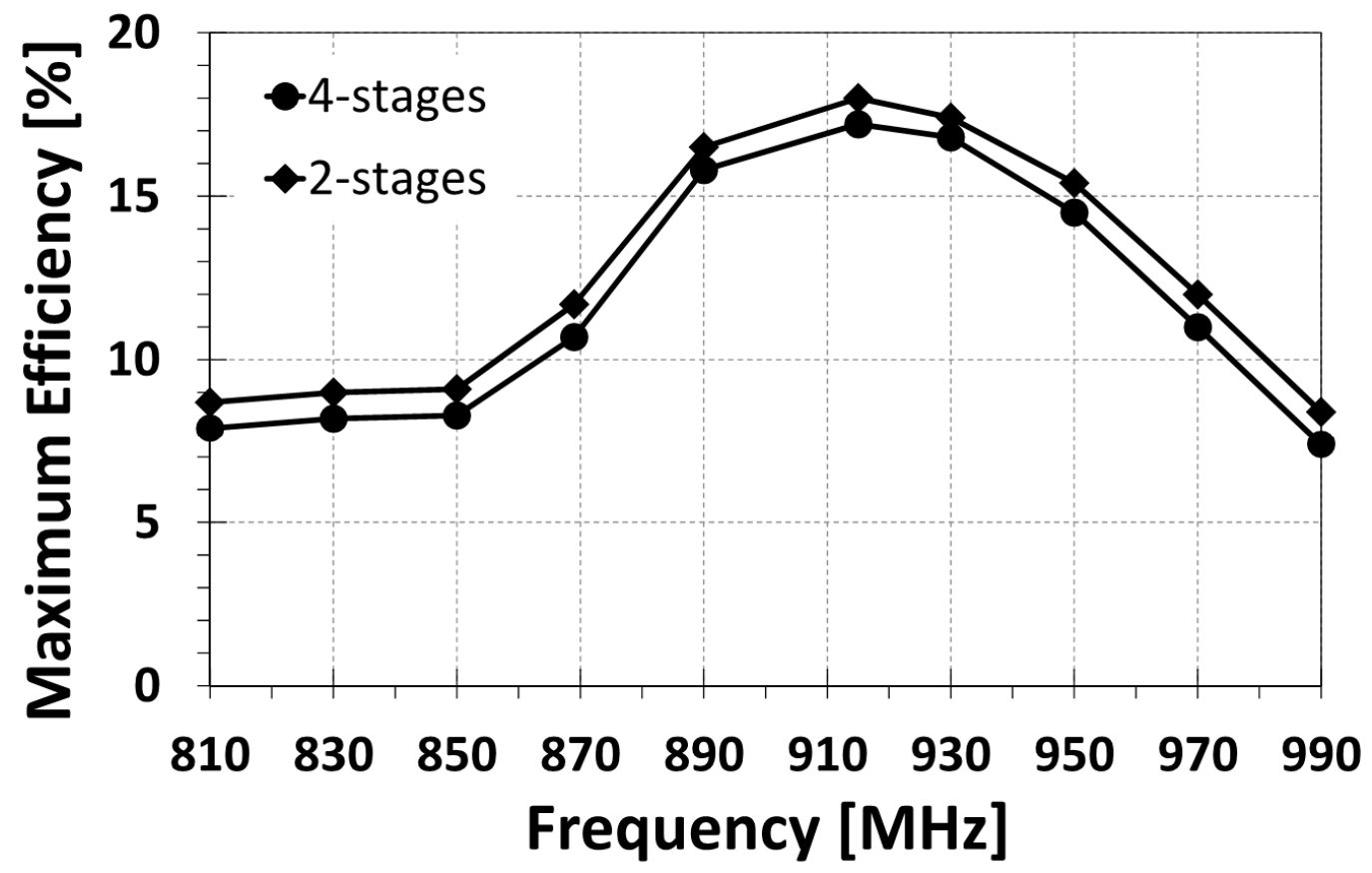

Figure 14. Maximum efficiency of RF energy harvesting system plotted vs. frequency for both four-stage and two-stage configurations. 
The system shows a higher efficiency at $915 \mathrm{MHz}$, which is due to the better impedance matching achieved at this frequency. The reconfigurable architecture adopted for the differential rectifier benefits the efficiency, which is quite high over a large $P_{I N}$ range.

The main characteristics of the whole system are summarized in Table 1, where the achieved results had been highlighted.

Table 1. Summary of the main characteristics of the whole system.

\begin{tabular}{|c|c|c|}
\hline Parameter & & Value \\
\hline RF-DC Converter Topology & & Differential \& Reconfigurable \\
\hline Technology & & CMOS ST130 nm \\
\hline Output Voltage & & $2.0 \mathrm{~V}$ \\
\hline Sensitivity & & -12.5 dBm@915 MHz \\
\hline RF Input Power Range & & $14 \mathrm{~dB}$ \\
\hline \multirow{4}{*}{ Maximum Efficiency } & \multirow{2}{*}{ 2-stages } & $18.0 \% @ 915 \mathrm{MHz}$ \\
\hline & & 11.7\%@869 MHz \\
\hline & \multirow{2}{*}{ 4-stages } & 17.2\%@915 MHz \\
\hline & & 10.7\%@869 MHz \\
\hline Bandwidth & 2-stages & $70 \mathrm{MHz}$ \\
\hline (with $\eta \geq 15 \%$ and centered $@ 915 \mathrm{MHz}$ ) & 4-stages & $55 \mathrm{MHz}$ \\
\hline Antenna Type & & Printed PCB Biconical Dipole \\
\hline \multirow{2}{*}{\multicolumn{2}{|c|}{ Antenna Gain }} & $1.6 \mathrm{dBi} @ 915 \mathrm{MHz}$ \\
\hline & & 1.4dBi@869 MHz \\
\hline
\end{tabular}

\section{Conclusions}

In this work, we have presented a RF energy harvester comprised of a RF-DC converter realized in ST130nm CMOS technology and a custom broadband PCB antenna with inductive coupling feeding. Prototypes of the system were realized by bonding on the custom antenna PCB the IC implementing the RF-DC converter and the smart voltage regulator.

The measurements performed by dedicated equipment have shown that the proposed RF energy harvesting system provides good performance at frequencies ( $869 \mathrm{MHz}$ and $915 \mathrm{MHz}$ ) compliant with regulations in force in North America and Europe. In addition, the reconfigurable architecture adopted for the converter allows for obtaining a regulated output voltage of $2 \mathrm{~V}$ over a $14 \mathrm{~dB}$ input power range, with a sensitivity of $-12.5 \mathrm{dBm}$ in case of RF power signal with carrier frequency of $915 \mathrm{MHz}$. Under these conditions, the conversion efficiency of the whole system remains higher than $10 \%$ for an input power range of more than $12 \mathrm{~dB}$, with a peak of $18 \%$.

\section{Author Contributions}

Alessandro Bertacchini, Luca Larcher and Stefano Scorcioni were responsible for the design and testing of the circuits and of the whole system described here. Moreno Maini and Luca Vincetti were responsible for the design and testing of the antenna described here as well as for the testing of the whole system. Alessandro Bertacchini, Luca Larcher and Luca Vincetti edited this paper after a review of all authors. 


\section{Conflicts of Interest}

The authors declare no conflict of interest.

\section{References}

1. FCC. $§ 15.247$ Operation within the Bands 902-928 MHz, 2400-2483.5 MHz, and 5725-5850 MHz. Available online: http://www.ecfr.gov/ (accessed on 13 March 2015).

2. ETSI EN 300 220-1 (V2.4.1). Electromagnetic compatibility and Radio spectrum Matters (ERM); Short Range Devices (SRD); Radio Equipment to be Used in the $25 \mathrm{MHz}$ to $1000 \mathrm{MHz}$ Frequency Range with Power Levels Ranging up to $500 \mathrm{~mW}$. Available online: http://www.etsi.org (accessed on 13 March 2015).

3. Yin, J.; Yi, J.; Law, M.K.; Ling, Y.; Lee, M.C.; Ng, K.P.; Gao, B.; Luong, H.C.; Bermak, A.; Chan, M.; et al. A system-on-chip EPC Gen-2 passive UHF RFID tag with embedded temperature sensor. IEEE J. Solid State Circuits 2010, 45, 2404-2420.

4. Le, T.; Mayaram, K.; Fiez, T. Efficient Far-Field Radio Frequency Energy Harvesting for Passively Powered Sensor Networks. IEEE J. Solid State Circuits 2008, 43, 1287-1302.

5. Chiu, H.; Lin, M.; Lin, C.; Ho, I.; Lin, W.; Fang, P.; Lee, Y.; Wen, Y.; Lu, S. Pain Control on Demand Based on Pulsed Radio-Frequency Stimulation of the Dorsal Root Ganglion Using a Batteryless Implantable CMOS SoC. IEEE Trans. Biomed. Circuits Syst. 2010, 4, 350-359.

6. Scorcioni, S.; Larcher, L.; Bertacchini, A. A Reconfigurable Differential CMOS RF Energy Scavenger With 60\% Peak Efficiency and $-21 \mathrm{dBm}$ Sensitivity. IEEE Microw. Wirel. Compon. Lett. 2013, 23, $155-157$.

7. Vincetti, L.; Maini, M.; Pinotti, E.; Larcher, L.; Scorcioni, S.; Bertacchini, A.; Grossi, D.; Tacchini, A. Broadband Printed Antenna for Radiofrequency Energy Harvesting. In Proceedings of the International Conference on Electromagnetics in Advanced Applications, Cape Town, South Africa, 2-7 September 2012; pp. 814-816.

8. Dickson, J.F. On-chip high-voltage generation in MNOS integrated circuits using an improved voltage multiplier technique. IEEE J. Solid State Circuits 1976, 11, 374-378.

9. Scorcioni, S.; Larcher, L.; Bertacchini, A.; Vincetti, L.; Maini, M. An integrated RF energy harvester for UHF wireless powering applications. In Proceedings of the IEEE Wireless Power Transfer Conference, Perugia, Italy, 15-16 May 2013; pp. 92-95.

10. Son, H.W.; Pyo, C.S. Design of RFID tag antennas using an inductively coupled feed. Electron. Lett. 2005, 41, 994-995.

11. Scorcioni, S.; Larcher, L.; Bertacchini, A. Optimized CMOS RF-DC converters for remote wireless powering of RFID applications. In Proceedings of the IEEE International Conference on RFID, Orlando, FL, USA, 3-5 April 2012; pp. 47-53.

12. Rao, K.V.S.; Nikitin, P.V.; Lam, S.F. Antenna design for UHF RFID tags: A review and a practical application. IEEE Trans. Antennas Propag. 2005, 53, 3870-3876. 
13. Scorcioni S.; Bertacchini A.; Larcher, L. A 868 MHz CMOS RF-DC power converter with $-17 \mathrm{dBm}$ input power sensitivity and efficiency higher than $40 \%$ over $14 \mathrm{~dB}$ input power range. In Proceedings of the IEEE 38th European Solid-State Circuits Conference (ESSCIRC), Palais des Congres Bordeaux, France 17-21 September 2012; pp. 109-112.

14. Balanis, C.A. Antenna Theory: Analysis and Design; Wiley: Hoboken, NJ, USA. 2005.

(C) 2015 by the authors; licensee MDPI, Basel, Switzerland. This article is an open access article distributed under the terms and conditions of the Creative Commons Attribution license (http://creativecommons.org/licenses/by/4.0/). 